\title{
STATISTICAL CHARACTERISTICS OF SOLAR PROTON FLARES
}

\author{
JIA-LONG WANG \\ Beijing Astronomical Observatory Chinese Academy of Sciences \\ Beijing 100080, China
}

\begin{abstract}
An investigation of the statistical behaviour of solar flares responsible for the proton events detected at the earth orbit would be of significance for solar physics and sun-earth research. Based on the data given by Kunches (1992) and other relevant data, we study the statistical behaviour of solar proton flares. The asymmetry of distributions, special property of hard X-ray bursts and relation to the large scale mean magnetic field of the proton flares are given in this paper.
\end{abstract}

\section{SPATIAL AND TEMPORAL DISTRIBUTIONS}

\section{Spatial Distribution}

Carrington longitudes of the proton flares whose locations and observed times are given in Kunches's list (1992) are calculated. It appears that proton flares occurred mainly in the longitude zone of $60^{\circ}-120^{\circ}$ and $300^{\circ}-360^{\circ}$ in Solar Cycle 21 , while in $60^{\circ}-120^{\circ}$ and $240^{\circ}-300^{\circ}$ for Solar Cycle 22 . It is obvious that the two active longitude zones are spaced by $180^{\circ}$ for the latter cycle.

The East-West asymmetry of solar flares affecting earth environment has been known for a long time. The helio-longitude of the proton flares studied shows that proton flares had an inhomogeneous distribution in their heliolongitudes, and the number of proton flares occurring at the west limb of the sun was two times the number at the east limb for 1976 to 1991. Also we find a variation of the East-West asymmetry of the proton flares with time for the period from 1976 to 1991 . Our study shows that the number of proton events in the north part of the sun is more than that in the south for both Solar Cycle 21 and Cycle 22.

\section{Temporal Distribution}

Based on Kunches's list, the yearly number of proton flares is studied. It is easy to find that the peak time of the number is later than that of the sunspot number for Cycle 21 for both the yearly value and the yearly smoothed value. For Solar Cycle 22, both peaks of numbers of the proton flares and sunspot numbers are in 1989 but the yearly smoothed peak of the proton flares is later than that of 
sunspot numbers. As we pointed out (Wang, 1989) that generally, solar activity indices vary with time unsynchronously.

We find that for the period from 1976 to 1991 , yearly number of the intense proton events and that of the weak proton events had a similar time-profile in contrast with the yearly distribution of intense hard X-ray bursts which is different from the distribution of weak hard X-ray bursts.

\section{THE RELEVANT HARD X-RAY BURSTS}

Generally, a solar proton flare would be accompanied by an intense hard X-ray burst. In trying to find the difference between hard X-ray bursts associated with solar proton events and hard X-ray bursts unassociated with solar proton events based on data of NASA T-M 4332 (Dennis et al., 1991), NASA T-M 4342-4344 (Dennis et al., 1992) and Kunches's table, 22 hard X-ray bursts associated with proton events and without $\gamma$-ray emissions and 46 hard X-ray bursts unassociated with proton events are studied, statistically. For these 68 bursts we obtain their ascending time $\left(\mathrm{T}_{a}\right)$, peak counting rates (Peak Rate), and maximum energies in $\mathrm{keV}$ (MaxkeV) from the data mentioned above. In Figure 1 the relation

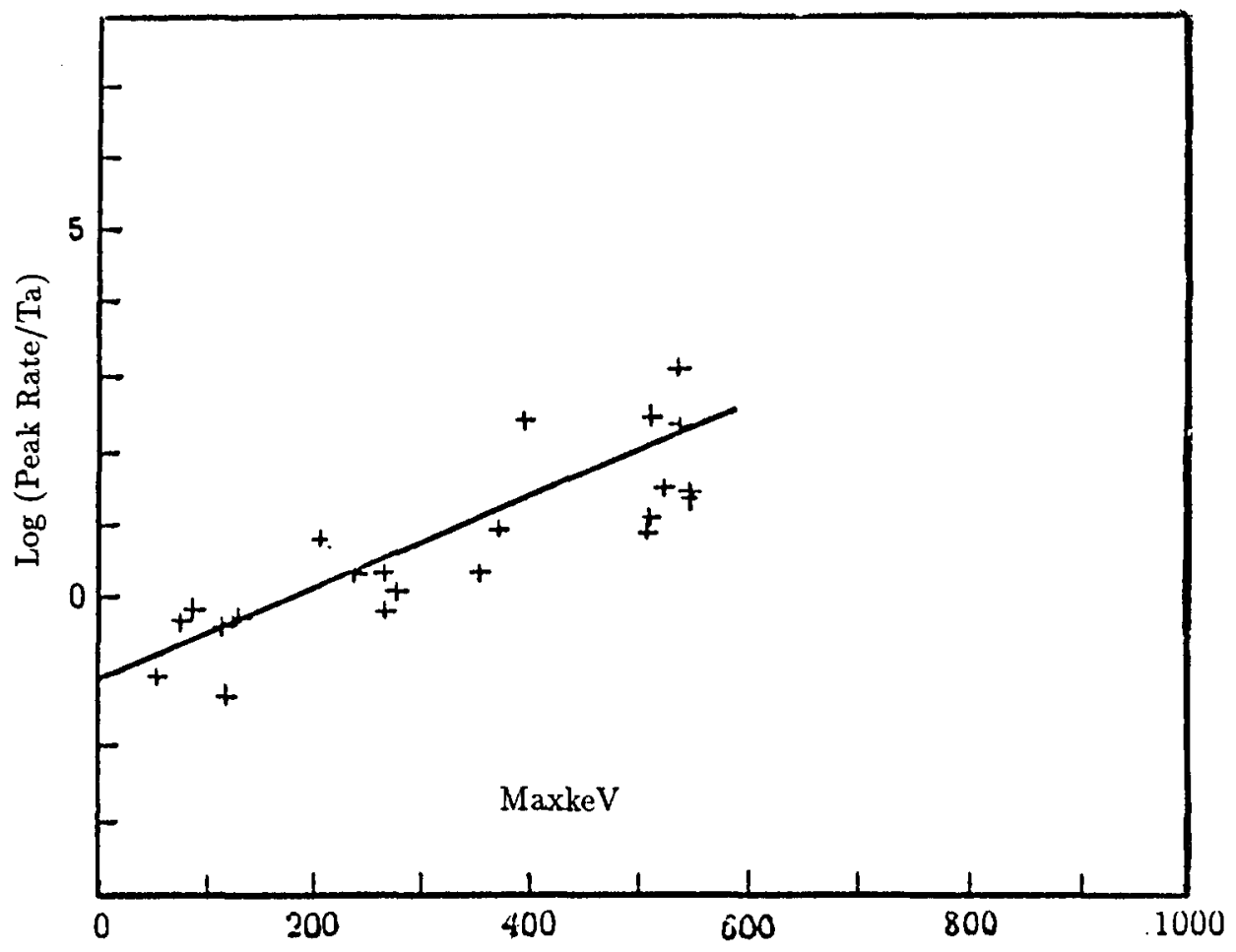

Fig.1. Hard X-ray bursts(1980-1989) associated with solar proton events 
of $\log \left(\right.$ Peak Rate/ $\left.\mathrm{T}_{a}\right)$ to $\mathrm{MaxkeV}$ for the 22 flares is given. We see in Figure 1 the good linear relation of $\log \left(\right.$ Peak Rate/ $\left.T_{a}\right)$ to MaxkeV. The corelation coefficient is about 0.847 much more than the critical coefficient, $0.423(\alpha=5 \%)$. The equation describing the relation is

$$
100 \log \left(\text { PeakRate } / T_{a}\right)=0.545 \mathrm{MaxkeV}-114
$$

While no such a relation is found for the other 46 hard X-ray bursts unassociated with proton events.

\section{THE RELATION TO THE SOLAR LARGE SCALE MAGNETIC FIELD}

It has long been known that conditions at the solar surface and in the interplanetary space play a great role in determining whether the proton flux produced by a flare can be detected by an artificial satellite of the earth or not. Using the solar magnetic field synoptic map published in Solar Geophysical Data, we divide the proton flares into two groups according to whether the location of the flare and solar disk center are on the same side of the neutral line of the solar large scale magnetic field or not. Then the distribution of the number of proton flares with the angle distance between the proton flare and solar disk center is investigated, and the result shows that most of the proton flares are located on the same side of the neutral line with the solar disk center, and the peak of the distribution is in the range of the angle distance of $20^{\circ}-50^{\circ}$.

\section{CONCLUSIONS}

1. The active longitude regions for 21 st and 22nd Solar Cycles are $60^{\circ}-120^{\circ}$ and $300^{\circ}-360^{\circ}$.

2. The number of proton flares occurring at west limb of the sun is two times the number at east limb of the sun for 1976-1991.

3. The west-east asymmetry and north-south asymmetry of proton flares changed with time obviously for 1976-1991.

4. It is found that for hard $\mathrm{X}$-ray bursts associated with proton flares and unassociated with $\gamma$-ray emissions, there is a good linear relation between $\log$ (Peak Rate/ $\mathrm{T}_{a}$ ) and MaxkeV.

5. Most of the proton flares and solar disk center in 1988-1991 were located on the same side of the neutral line of solar large scale mean magnetic field.

This work is supported by NNSF of China.

\section{REFERENCES}

Dennis, B. R. et al., 1991, NASA Technical Memorandum 4332, NASA, USA.

Dennis, B, R. et al., 1992. NASA Technical Memorandum 4342, 4343, 4344, NASA, USA.

Kunches, J. 1992, SESC PRF, 858, 25.

Wang, J. L., 1989, Publ. Yunnan Obs., Special Issue I, 330. 